\title{
A Chromosome-Level Genome Assembly of the Reed Warbler (Acrocephalus scirpaceus)
}

\author{
Camilla Lo Cascio Sætre ${ }^{1, *}$, Fabrice Eroukhmanoff ${ }^{1}$, Katja Rönkä2,3, Edward Kluen ${ }^{2,3}$, Rose Thorogood ${ }^{2,3}$, \\ James Torrance ${ }^{4}$, Alan Tracey ${ }^{4}$, William Chow ${ }^{4}$, Sarah Pelan ${ }^{4}$, Kerstin Howe ${ }^{4}$, Kjetill S. Jakobsen ${ }^{1}$, and \\ Ole K. Tørresen ${ }^{1}$ \\ ${ }^{1}$ Centre for Ecological and Evolutionary Synthesis, University of Oslo, Norway \\ ${ }^{2}$ HiLIFE Helsinki Institute of Life Sciences, University of Helsinki, Finland \\ ${ }^{3}$ Research Programme in Organismal and Evolutionary Biology, Faculty of Biological and Environmental Sciences, University of Helsinki, Finland \\ ${ }^{4}$ Tree of Life, Wellcome Sanger Institute, Cambridge, United Kingdom \\ *Corresponding author: E-mail: c.l.c.satre@ibv.uio.no.
}

Accepted: 6 September 2021

\begin{abstract}
The reed warbler (Acrocephalus scirpaceus) is a long-distance migrant passerine with a wide distribution across Eurasia. This species has fascinated researchers for decades, especially its role as host of a brood parasite, and its capacity for rapid phenotypic change in the face of climate change. Currently, it is expanding its range northwards in Europe, and is altering its migratory behavior in certain areas. Thus, there is great potential to discover signs of recent evolution and its impact on the genomic composition of the reed warbler. Here, we present a high-quality reference genome for the reed warbler, based on PacBio, 10x, and $\mathrm{Hi}-\mathrm{C}$ sequencing. The genome has an assembly size of 1,075,083,815 bp with a scaffold N50 of 74,438,198 bp and a contig N50 of 12,742,779 bp. BUSCO analysis using aves_odb10 as a model showed that $95.7 \%$ of BUSCO genes were complete. We found unequivocal evidence of two separate macrochromosomal fusions in the reed warbler genome, in addition to the previously identified fusion between chromosome Z and a part of chromosome 4A in the Sylvioidea superfamily. We annotated 14,645 protein-coding genes, and a BUSCO analysis of the protein sequences indicated $97.5 \%$ completeness. This reference genome will serve as an important resource, and will provide new insights into the genomic effects of evolutionary drivers such as coevolution, range expansion, and adaptations to climate change, as well as chromosomal rearrangements in birds.
\end{abstract}

Key words: genome assembly, Hi-C sequencing, long reads, reference genome, Acrocephalus scirpaceus. .

\section{Significance}

The reed warbler (Acrocephalus scirpaceus) has been lacking a genomic resource, despite having been broadly researched in studies of coevolution, ecology, and adaptations to climate change. Here, we generated a chromosome-length genome assembly of the reed warbler, and found evidence of macrochromosomal fusions in its genome, which are likely of recent origin. This genome will provide the opportunity for a deeper understanding of the evolution of genomes in birds, as well as the evolutionary path and possible future of the reed warbler.

\section{Introduction}

The ecology and evolution of the reed warbler (Acrocephalus scirpaceus) has been of interest for over 40 years (Thorogood et al. 2019) as it is one of the favorite host species of the brood- parasitic common cuckoo (Cuculus canorus) (Davies and Brooke 1989; Stokke et al. 2018). Decades of field experiments have demonstrated behavioral coevolution and spatial and temporal variation in species interactions (e.g., Thorogood

(C) The Author(s) 2021. Published by Oxford University Press on behalf of the Society for Molecular Biology and Evolution.

This is an Open Access article distributed under the terms of the Creative Commons Attribution License (https://creativecommons.org/icenses/by/4.0/), which permits unrestricted reuse, distribution, and reproduction in any medium, provided the original work is properly cited. 
and Davies 2013). However, the reed warbler's response to climate change has begun to attract increasing attention. Reed warblers are experiencing far less severe declines in population size than is typical for long-distance migrants (Both et al. 2010; Vickery et al. 2014). In fact, they are expanding their breeding range northwards into Fennoscandia (Järvinen and Ulfstrand 1980; Røed 1994; Stolt 1999; Brommer et al. 2012), and have generally increased their productivity following the rise in temperature (Schaefer et al. 2006; Eglington et al. 2015; Meller et al. 2018). They are also showing rapid changes in phenology (Halupka et al. 2008), and migratory behavior; instead of crossing the Sahara, monitoring suggests that some reed warblers now remain on the Iberian Peninsula over winter (Chamorro et al. 2019). Morphological traits such as body mass and wing shape have been shown to change rapidly in reed warbler populations, indicating possible local adaptation (Kralj et al. 2010; Salewski et al. 2010; Sætre et al. 2017). Genetic differentiation is generally low between reed warbler populations, but moderate levels of differentiation have been connected to both migratory behavior (Procházka et al. 2011) and wing shape (Kralj et al. 2010). Reed warblers thus provide a promising system to study population, phenotypic, and genetic responses to climate change.

Although there has been an increasing number of avian genome assemblies in recent years (e.g., Feng et al. 2020), many nonmodel species, including the reed warbler, are still lacking a genome resource. To date, the closest relative to the reed warbler with a published reference genome is the great tit (Parus major) (GCA_001522545.3, deposited in NCBI; Laine et al. 2016), but the unpublished genome of the garden warbler (Sylvia borin) is available in public databases (GCA_014839755.1, deposited in NCBI). There is also a genome in preprint from the Acrocephalus genus, the great reed warbler ( $A$. arundinaceus) (Sigeman, Strandh, et al. 2020), but the scaffolds are not chromosome length.

Here, we present the first genome assembly of the reed warbler, based on PacBio, 10x, and $\mathrm{Hi}-\mathrm{C}$ sequencing, with descriptions of the assembly, manual curation, and annotation. This genome will be a valuable resource for a number of studies, including studies of coevolution, population genomics, adaptive evolution, and comparative genomics. For reducedrepresentation sequencing (e.g., RAD-seq) studies, it will help produce a more robust SNP set than with a de novo approach (Shafer et al. 2017). It will facilitate the detection of selective sweeps, and provide the physical localization of variants (Manel et al. 2016), thus giving insight into the potential genes involved in adaptation. Furthermore, the genome will be an important resource in the study of chromosomal rearrangements in birds.

\section{Results and Discussion}

\section{Genome Assembly and Genome Quality Evaluation}

We generated $3,810,665$ reads with PacBio, with an average read length of $16 \mathrm{~kb}$ at $61 \times$ coverage. We further obtained
$277,617,608$ paired-end reads $(2 \times 150)$ with $10 \times$ Genomics, and $185,974,525$ paired-end reads $(2 \times 150)$ with $\mathrm{Hi}-\mathrm{C}$, at $83 \times$ and $56 x$ coverage, respectively. The final genome assembly was $1.08 \mathrm{~Gb}$ in length, and contains 1,081 contigs (contig N50 of $13 \mathrm{Mb}$ ) and 200 scaffolds (scaffold N50 of $74 \mathrm{Mb}$ ) (table 1). The completeness of the assembled genome is high: of the 8,338 universal avian single-copy orthologs, we identified 7,978 complete BUSCOs (95.7\%), including 7,920 single-copy (95.0\%), and 58 duplicated BUSCOs (0.7\%). Fifty-nine BUSCOs (0.7\%) were fragmented, and 301 BUSCOs (3.6\%) were missing.

\section{Genome Annotation}

The GC content of the reed warbler genome assembly was $41.9 \%$. The total repeat content of the assembly was $10.94 \%$, with LTR elements as the most common type of repeat (4.50\%) followed by LINEs (4.11\%) (table 1 ).

Using the Comparative Annotation Toolkit, based on a whole-genome multiple alignments from Cactus, we predicted 14,645 protein coding genes, with an average Coding DNA Sequence (CDS) length of 1,782 bp, and an average intron length of $2,918 \mathrm{bp}$ (table 1). The annotated genes had $97.5 \%$ completeness (based on predicted proteins).

\section{Synteny Analysis}

The reed warbler genome showed high synteny with the great tit genome, though with some notable differences (fig. 1). The reed warbler chromosome 6 is a fusion of great tit chromosomes 7 and 8 , and reed warbler chromosome 8 is a fusion of great tit chromosomes 6 and 9. Interestingly, these chromosomes are not fused in the garden warbler genome (supplementary fig. 1, Supplementary Material online), but correspond to the great tit chromosomes. This suggests that the fusions evolved relatively recently, perhaps at the base of the Acrocephalidae branch within Sylvioidea, but further research is needed to determine this. Hi-C contact maps confirm that the chromosomes assembled in the reed warbler genome are unbroken (supplementary fig. 2, Supplementary Material online). Interchromosomal rearrangements are rare in avian evolution (Ellegren 2010; Skinner and Griffin 2012), with some exceptions, such as in the orders Falconiformes (Damas et al. 2017) and Psittaciformes (Furo et al. 2018). In fact, in all or most species of Psittaciformes, chicken chromosomes 6 and 7, and 8 and 9 are fused (Furo et al. 2018; Kretschmer et al. 2018)—the same chromosomes involved in the fusions discovered in the reed warbler genome. We can only speculate about the significance of this without more data. Passeriformes, the sister group of Psittaciformes, exhibit much lower rates of interchromosomal rearrangements, despite being a large, highly diverse order (Kretschmer et al. 2021). There is still a large knowledge gap in the cytogenetics of birds (Degrandi et al. 2020), and more 
Table 1

Summary Statistics of the Reed Warbler Genome Assembly and Annotation

\begin{tabular}{|c|c|c|c|}
\hline \multicolumn{4}{|l|}{ Genome Assembly } \\
\hline & Estimated genome size & $1.13 \mathrm{~Gb}$ & \\
\hline & Guanine and cytosine content & $41.91 \%$ & \\
\hline & N50 length (contig) & $13 \mathrm{Mb}$ & \\
\hline & Longest contig & $48 \mathrm{Mb}$ & \\
\hline & Total length of contigs & $1.07 \mathrm{~Gb}$ & \\
\hline & N50 length (scaffold) & $74.44 \mathrm{Mb}$ & \\
\hline & Longest scaffold & $153.80 \mathrm{Mb}$ & \\
\hline & Total length of scaffolds & $1.08 \mathrm{~Gb}$ & \\
\hline & Complete BUSCOs & $95.7 \%$ & \\
\hline \multirow[t]{8}{*}{ Transposable Elements } & & Percent (\%) & Total length \\
\hline & DNA & 0.22 & $2.35 \mathrm{Mb}$ \\
\hline & LINE & 4.11 & $44.2 \mathrm{Mb}$ \\
\hline & SINE & 0.09 & $0.98 \mathrm{Mb}$ \\
\hline & LTR & 4.50 & $48.4 \mathrm{Mb}$ \\
\hline & Unknown & 0.55 & $5.9 \mathrm{Mb}$ \\
\hline & $\begin{array}{l}\text { Other (satellites, simple repeats, } \\
\text { low complexity) }\end{array}$ & 1.49 & $16 \mathrm{Mb}$ \\
\hline & Total & 10.94 & $117.6 \mathrm{Mb}$ \\
\hline \multicolumn{4}{|l|}{ Protein-Coding Genes } \\
\hline & Predicted genes & 14,645 & \\
\hline & $\begin{array}{l}\text { Average coding sequence length } \\
\text { (bp) }\end{array}$ & 1,782 & \\
\hline & Average exon length (bp) & 284 & \\
\hline & Average intron length (bp) & 2918 & \\
\hline & Complete BUSCOs & $97.5 \%$ & \\
\hline
\end{tabular}

research is needed to determine the rarity of the fusions we discovered in the reed warbler genome.

We furthermore confirm the previously identified neo-sex chromosome (Pala et al. 2012; Sigeman, Ponnikas, et al. 2020), a fusion between the ancestral chromosome $Z$ and a part of chromosome 4A (according to chromosome naming from the zebra finch). This fusion is thought to have occurred at the base of the Sylvioidea branch (Pala et al. 2012) and is shared with all species of Sylvioidea studied so far (Sigeman, Ponnikas, et al. 2020). Figure 1 clearly shows that reed warbler chromosome Z corresponds to great tit chromosome Z, plus a part of great tit chromosome 4A, whereas reed warbler chromosome Z corresponds to garden warbler chromosome Z (supplementary fig. 1, Supplementary Material online).

\section{Conclusion}

In this study, we present the first assembled and annotated genome for the reed warbler $A$. scirpaceus. We have accomplished this through utilizing long read PacBio sequencing, and scaffolding with paired-end $10 \times$ and $\mathrm{Hi}-\mathrm{C}$ reads. In addition to the previously identified autosomesex chromosome fusion shared by all members of
Sylvioidea, we found unequivocal evidence of two novel macrochromosomal fusions in the reed warbler genome. Further research is needed to determine the evolutionary age of these fusions, especially because they are not present in the garden warbler genome, suggesting they are relatively new. This genome will serve as an important resource to increase our knowledge of chromosomal rearrangements in birds, both their prevalence and their significance for avian evolution. Furthermore, the genome will, through the identification of genetic variants and information of the function of associated genes, provide a deeper insight into the evolution of the reed warbler, a bird which will continue to fascinate researchers for years to come.

\section{Materials and Methods}

\section{Sampling and Isolation of Genomic DNA}

Blood was collected from a brachial vein of a female reed warbler (subspecies $A$. scirpaceus scirpaceus, $\mathrm{NCBI}$ Taxonomy ID: 126889) in Storminnet, Porvoo $\left(60^{\circ} 19^{\prime 2} 24.9^{\prime \prime} \mathrm{N}, 25^{\circ} 35^{\prime 2} 23.0^{\prime \prime} \mathrm{E}\right)$, Finland, on May 22, 2019. 


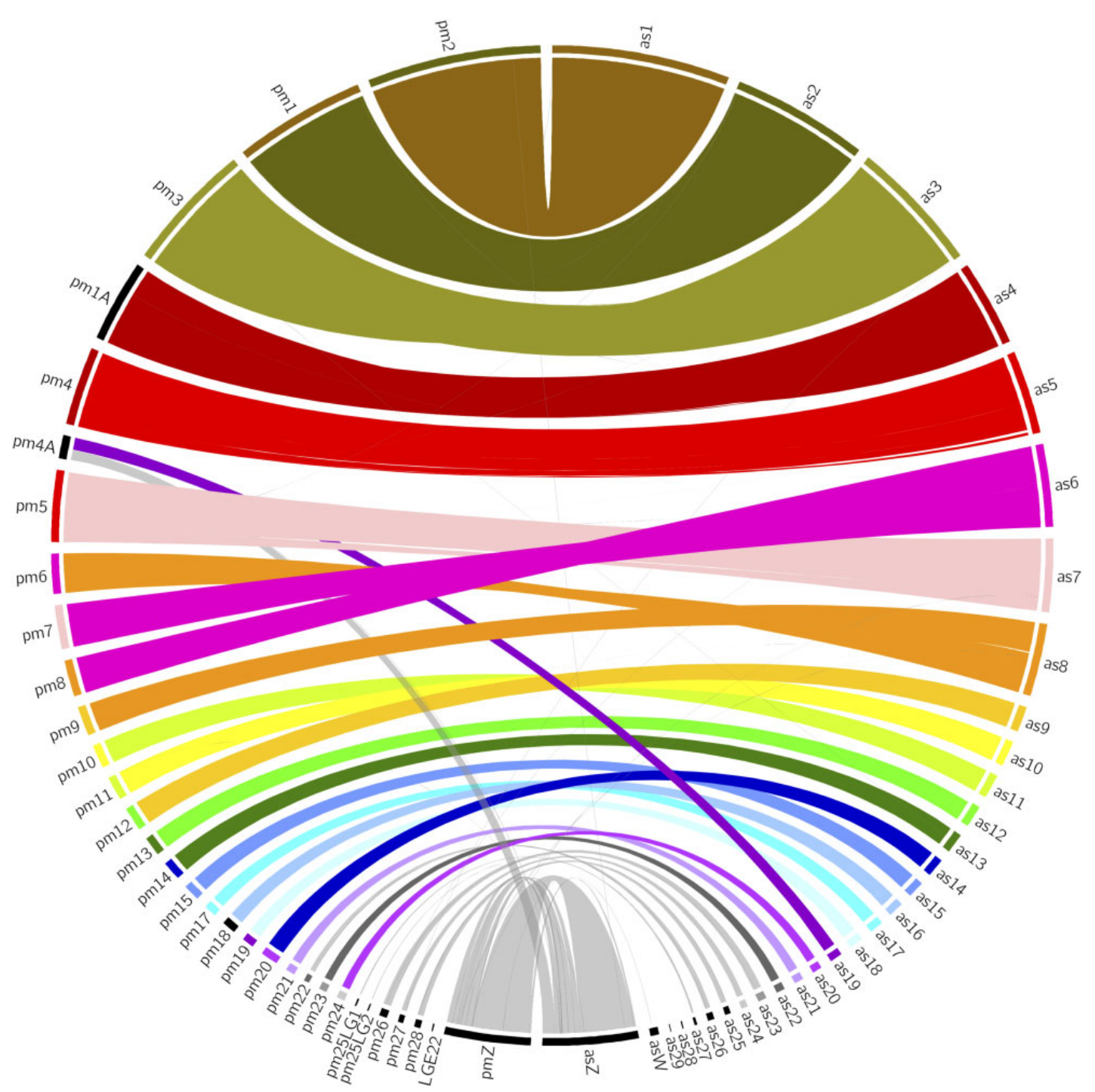

Fig. 1.-Circos plot showing the synteny between the reed warbler (on the right side, denoted with the prefix as [Acrocephalus scirpaceus]) and the great tit (left side, prefix pm [Parus major]) genome assemblies. The reed warbler chromosome 6 is a fusion of great tit chromosomes 7 and 8, whereas reed warbler chromosome 8 is a fusion of great tit chromosomes 6 and 9 (see Hi-C contact maps in supplementary fig. 2, Supplementary Material online). The reed warbler chromosome $Z$ corresponds to great tit chromosome $Z$, and a part of great tit chromosome 4 A.

Catching and sampling procedures complied with the Finnish law on animal experiments and permits were licensed by the National Animal Experiment Board (ESAVI/3920/2018) and Southwest Finland Regional Environment Centre (VARELY/ 758/2018). Reed warblers were trapped with a mist net, ringed and handled by E.K. under his ringing license.

The blood ( $80 \mathrm{ml}$ ) was divided and stored separately in $500 \mathrm{ml}$ ethanol, and in $500 \mathrm{ml}$ SET buffer $(0.15 \mathrm{M} \mathrm{NaCl}$, 0.05M Tris, 0.001M EDTA, pH 8.0). The samples were immediately placed in liquid nitrogen, and kept at $-80{ }^{\circ} \mathrm{C}$ when stored. We performed phenol-chloroform DNA isolation on the sample stored in SET buffer, following a modified protocol from Sambrook et al. (1989).

\section{Library Preparation and Sequencing}

DNA quality was checked using a combination of a fluorometric (Qubit, Invitrogen), UV absorbance (Nanodrop, Thermo Fisher) and DNA fragment length assays (HS-50 kb fragment kit from AATI, now part of Agilent Inc.). The PacBio library was prepared using the Pacific Biosciences Express library preparation protocol. DNA was fragmented to $35 \mathrm{~kb}$. Size selection of the final library was performed using BluePippin with a $15 \mathrm{~kb}$ cut-off. Six single-molecule realtime (SMRT) cells were sequenced using Sequel Polymerase v3.0 and Sequencing chemistry v3.0 on a PacBio RS II instrument. The $10 \times$ Genomics Chromium linked-read protocol $(10 \times$ Genomics Inc.) was used to prepare the $10 \times$ library, 
and due to the reed warbler's smaller sized genome, only $0.7 \mathrm{ng} / \mu \mathrm{l}$ of high molecular weight DNA was used as input. A high-throughput chromosome conformation capture ( $\mathrm{Hi}-\mathrm{C})$ library was constructed using $50 \mu$ l of blood, following step 10 and onwards in the Arima Hi-C (Arima Genomics) library protocol for whole blood. Adaptor ligation with Unique dual indexing (Illumina) were chosen to match the indexes from the $10 \times$ linked-read library for simultaneous paired-end sequencing (150 bp) on the same lane on an Illumina HiSeq $X$ platform. Both libraries were quality controlled using a Fragment analyzer NGS kit (AATI) and qPCR with the Kapa library quantification kit (Roche) prior to sequencing.

The sequencing was provided by the Norwegian Sequencing Centre (https://www.sequencing.uio.no, last accessed September 17, 2021), a national technology platform hosted by the University of Oslo and supported by the "Functional Genomics" and "Infrastructure" programs of the Research Council of Norway and the South-Eastern Regional Health Authorities.

\section{Genome Size Estimation and Genome Assembly}

The genome size of the reed warbler was estimated by a kmer analysis of $10 \times$ reads using Jellyfish v. 2.3.0 (Marçais and Kingsford 2011) and Genome Scope v. 1.0 (Vurture et al. 2017), with a k-mer size of 21 . The estimated genome size was 1,130,626,830 bp.

We assembled the long-read PacBio sequencing data with FALCON and FALCON-Unzip (falcon-kit 1.5.2 and falconunzip 1.3.5) (Chin et al. 2016). Falcon was run with the following parameters: length_cutoff $=-1$; length_cutoff_pr $=$ 1000; pa_HPCdaligner_option $=-\mathrm{v}-\mathrm{B} 128-\mathrm{M} 24$; pa_daligner_option $=-\mathrm{e} 0.8-12000-\mathrm{k} 18-\mathrm{h} 480-\mathrm{w} 8-\mathrm{s} 100$; ovlp_HPCdaligner_option $=-v-B 128-M 24$; ovlp_daligner_option $=-k 24-\mathrm{e} .94-\mid 3000-\mathrm{h} 1024-\mathrm{s} 100 ;$ pa_DBsplit_option = $-x 500$-s200; ovlp_DBsplit_option $=-x 500-s 200$; falcon_sense_option $=$-output-multi - min-idt 0.70 -min-cov 3 -max-nread 200; overlap_filtering_setting $=-$ max-diff 100 -max-cov 100 -min-cov 2. Falcon-unzip was run with default settings. The purge_haplotigs pipeline v. 1.1.0 (Roach et al. 2018) was used to curate the diploid assembly, with -15, -m35, -h190 for the contig coverage, and -a60 for the purge pipeline. Next, we scaffolded the curated assembly with the $10 \times$ reads using Scaff $10 X$ v. 4.1 (https://github.com/wtsi-hpag/Scaff10X, last accessed September 17, 2021), and the Hi-C reads using SALSA v. 2.2 (Ghurye et al. 2017). Finally, we polished the assembly (combined with the alternative assembly from Falcon-Unzip), first with PacBio reads using pbmm2 v. 1.2.1, which uses minimap2 (Li 2018) internally (v. 2.17), and then with $10 \times$ reads for two rounds with Long Ranger v. 2.2.2 (Marks et al. 2019) and FreeBayes v. 1.3.1 (Garrison and Marth 2012).

\section{Curation}

The assembly was decontaminated and manually curated using the gEVAL browser (Chow et al. 2016; Howe et al. 2021), resulting in 521 corrections (breaks, joins and removal of erroneously duplicated sequence). HiGlass (Kerpedjiev et al. 2018) and PretextView (https://github.com/wtsi-hpag/ PretextView, last accessed September 17, 2021) were used to visualize and rearrange the genome using $\mathrm{Hi}-\mathrm{C}$ data, and PretextSnapshot (https://github.com/wtsi-hpag/ PretextSnapshot, last accessed September 17, 2021) was used to generate an image of the Hi-C contact map. The corrections made reduced the total length of scaffolds by $0.5 \%$ and the scaffold count by $44.6 \%$, and increased the scaffold N50 by $20.2 \%$. Curation identified and confirmed 29 autosomes and the $\mathrm{Z}$ and $\mathrm{W}$ chromosomes, to which $98.6 \%$ of the assembly sequences were assigned.

\section{Genome Quality Evaluation}

We assessed the quality of the assembly with the assemblathon_stats.pl script (Bradnam et al. 2013) and investigated the completeness of the genome with Benchmarking Universal Single-Copy Orthologs (BUSCO) v. 5.0.0 (Simão et al. 2015), searching for 8,338 universal avian single-copy orthologs (aves_odb10).

\section{Genome Annotation}

We used a repeat library provided by Alexander Suh called bird_library_250ct2020 and described in Peona et al. (2020) to softmask repeats in the reed warbler genome assembly. Softmasked genome assemblies for golden eagle (Aquila chrysaetos), chicken (Gallus gallus), great tit (Parus major), Anna's hummingbird (Calypte anna), zebra finch (Taeniopygia guttata), great reed warbler (Acrocephalus arundinaceus), icterine warbler (Hippolais icterina), collared flycatcher (Ficedula albicollis), and New Caledonian crow (Corvus moneduloides) were downloaded from NCBI. The triangle subcommand from Mash v. 2.3 (Ondov et al. 2016) was used to estimate a lower-triangular distance matrix, and a Python script (https://github.com/marbl/Mash/issues/9\#issuecomment-509837201, last accessed September 17, 2021) was used to convert the distance matrix into a full matrix. The full matrix was used as input to RapidNJ v. 2.3.2 (Simonsen et al. 2008) to create a guide tree based on the neighbor-joining method. Cactus v. 1.3.0 (Armstrong et al. 2020) was run with the guide tree and the softmasked genome assemblies as input.

We also downloaded the annotation for chicken, and used it as input to the Comparative Annotation Toolkit (CAT) v. 2.2.1-36-gfc1623d (Fiddes et al. 2018) together with the hierarchical alignment format file from Cactus. Chicken was used as reference genome, reed warbler as the target genome and the AUGUSTUS (Stanke et al. 2008) species parameter was set to "chicken." InterProScan v. 5.34-73 (Jones et al. 2014) was run on the predicted proteins to find functional annotations, and DIAMOND v. 2.0.7 (Buchfink et al. 2021) was used to compare the predicted proteins against 
UniProtKB/Swiss-Prot release 2021_03 (The UniProt Consortium 2021). AGAT v. 0.5.1 (Dainat 2021) was used to generate statistics from the GFF3 file with annotations and to add functional annotations from InterProScan and gene names from UniProtKB/Swiss-Prot. BUSCO v. 5.0.0 was used to assess the completeness of the annotation.

\section{Synteny Analysis}

We aligned the assembly against the great tit (Parus major) and the garden warbler (Sy/via borin) genome assemblies with minimap2 v. 2.18-r1015 and extracted only alignments longer than 5,000 bp. The bundlelinks from circos-tools v. 0.23 was used to merge neighboring links using default options and a plot was created using circos v. 0.69-8.

\section{Supplementary Material}

Supplementary data are available at Genome Biology and Evolution online.

\section{Acknowledgments}

We would like to thank Marjo Saastamoinen, Suvi Sallinen, Paolo Momigliano, and the Molecular Ecology and Systematics laboratory in the University of Helsinki for facilitating DNA extraction. We would like to thank Ave ToomingKlunderud and the Norwegian Sequencing Centre for performing the sequencing. We also thank Pasi Rastas from the HiLIFE BioData Analytics Service unit, for his assistance with preliminary analyses. The computations were performed on resources provided by UNINETT Sigma2 - the National Infrastructure for High Performance Computing and Data Storage in Norway. This work was supported by Research Council of Norway by grant numbers 251076 and 300032 to K.S.J. and a HiLIFE Start-up grant and a University of Helsinki Faculty of Biological and Environmental Sciences travel grant to R.T.

\section{Author Contributions}

C.L.C.S., F.E., K.R., K.S.J., O.K.T., and R.T. designed the research. E.K., K.R., and R.T. collected the sample. K.R. extracted DNA. C.L.C.S. and O.K.T. performed the research and/or analyzed the data. A.T., J.T., K.H., S.P., and W.C. Curated the assembly. C.L.C.S. drafted the manuscript. All authors read and approved the final manuscript.

\section{Data Availability}

The reference genome of Acrocephalus scirpaceus (bAcrSci1), and the raw sequence data, have been deposited in the European Nucleotide Archive under the BioProject number PRJEB45715. Genome annotations are available at Figshare and can be accessed at https://doi.org/10.6084/m9.figshare. 16622302.v1.

\section{Literature Cited}

Armstrong J, et al. 2020. Progressive Cactus is a multiple-genome aligner for the thousand-genome era. Nature 587(7833):246-251.

Both C, et al. 2010. Avian population consequences of climate change are most severe for long-distance migrants in seasonal habitats. Proc Biol Sci. 277(1685):1259-1266.

Bradnam KR, et al. 2013. Assemblathon 2: evaluating de novo methods of genome assembly in three vertebrate species. Gigascience 2(1):10.

Brommer JE, Lehikoinen A, Valkama J. 2012. The breeding ranges of central European and arctic bird species move poleward. PLOS One 7(9):e43648.

Buchfink B, Reuter K, Drost HG. 2021. Sensitive protein alignments at tree-of-life scale using DIAMOND. Nat Methods 18(4):366-368.

Chamorro D, Nieto I, Real R, Muñoz AR. 2019. Wintering areas on the move in the face of warmer winters. Ornis Fennica 96:41-54.

Chin CS, et al. 2016. Phased diploid genome assembly with single molecule real-time sequencing. Nat Methods 13(12):1050-1054.

Chow W, et al. 2016. gEVAL-a web-based browser for evaluating genome assemblies. Bioinformatics 32(16):2508-2510.

Dainat J. 2021. AGAT: Another Gff Analysis Toolkit to Handle Annotations in Any GTF/GFF Format (Version v0.5.1). Zenodo. Available from: https://www.doi.org/10.5281/zenodo.3552717. Accessed September 17, 2021.

Damas J, et al. 2017. Upgrading short-read animal genome assemblies to chromosome level using comparative genomics and a universal probe set. Genome Res. 27(5):875-884.

Davies NB, Brooke ML. 1989. An experimental study of co-evolution between the cuckoo, Cuculus canorus, and its hosts. I. Host egg discrimination. J Anim Ecol. 58(1):207-224.

Degrandi TM, et al. 2020. Introducing the bird chromosome database: an overview of cytogenetic studies in birds. Cytogenet Genome Res. 160(4):199-205.

Eglington SM, et al. 2015. Latitudinal gradients in the productivity of European migrant warblers have not shifted northwards during a period of climate change. Global Ecol Biogeogr. 24(4):427-436.

Ellegren H. 2010. Evolutionary stasis: the stable chromosomes of birds. Trends Ecol Evol. 25(5):283-291.

Feng S, et al. 2020. Dense sampling of bird diversity increases power of comparative genomics. Nature 587(7833):252-257.

Fiddes IT, et al. 2018. Comparative Annotation Toolkit (CAT) - simultaneous clade and personal genome annotation. Genome Res. 28(7):1029-1038.

Furo IDO, et al. 2018. Chromosome painting in Neotropical long- and short-tailed parrots (Aves, Psittaciformes): phylogeny and proposal for a putative ancestral karyotype for tribe Arini. Genes 9: 491. Available from: 10.3390/genes9100491. Accessed September 17, 2021.

Garrison E, Marth G. 2012. Haplotype-based variant detection from shortread sequencing. arXiv Preprint. Available from: https://arxiv.org/abs/ 1207.3907. Accessed September 17, 2021.

Ghurye J, Pop M, Koren S, Bickhart D, Chin CS. 2017. Scaffolding of long read assemblies using long range contact information. BMC Genomics 18(1):527.

Halupka L, Dyrcz A, Borowiec M. 2008. Climate change affects breeding of reed warblers Acrocephalus scirpaceus. J Avian Biol. 39(1):95-100.

Howe K, et al. 2021. Significantly improving the quality of genome assemblies through curation. GigaScience 10(1): giaa153.

Järvinen O, Ulfstrand S. 1980. Species turnover of a continental bird fauna: northern Europe, 1850-1970. Oecologia 46(2):186-195. 
Jones $P$, et al. 2014. InterProScan 5: genome-scale protein function classification. Bioinformatics 30(9):1236-1240.

Kerpedjiev P, et al. 2018. HiGlass: web-based visual exploration and analysis of genome interaction maps. Genome Biol. 19(1):125.

Kralj J, Procházka P, Fainová D, Patzenhauerová H, Tutiš V. 2010. Intraspecific variation in the wing shape and genetic differentiation of reed warblers Acrocephalus scirpaceus in Croatia. Acta Ornithol. 45(1):51-58.

Kretschmer R, Ferguson-Smith MA, de Oliveira EHC. 2018. Karyotype evolution in birds: from conventional staining to chromosome painting. Genes 9:181.

Kretschmer R, et al. 2021. Karyotype evolution and genomic organization of repetitive DNAs in the saffron finch, Sicalis flaveola (Passeriformes, Aves). Animals 11(5):1456. Available from: 10.3390/ani11051456. Accessed September 17, 2021.

Laine VN, Great Tit HapMap Consortium, et al. 2016. Evolutionary signals of selection on cognition from the great tit genome and methylome. Nat Commun. 7:10474.

Li H. 2018. Minimap2: pairwise alignment for nucleotide sequences. Bioinformatics 34(18):3094-3100.

Manel S, et al. 2016. Genomic resources and their influence on the detection of the signal of positive selection in genome scans. Mol Ecol. 25(1):170-184.

Marçais G, Kingsford C. 2011. A fast, lock-free approach for efficient parallel counting of occurrences of k-mers. Bioinformatics 27(6):764-770.

Marks $P$, et al. 2019. Resolving the full spectrum of human genome variation using linked-reads. Genome Res. 29(4):635-645.

Meller K, Piha M, Vähätalo AV, Lehikoinen A. 2018. A positive relationship between spring temperature and productivity in 20 songbird species in the boreal zone. Oecologia 186(3):883-893.

Ondov BD, et al. 2016. Mash: fast genome and metagenome distance estimation using MinHash. Genome Biol. 17(1):132.

Pala I, et al. 2012. Evidence of a neo-sex chromosome in birds. Heredity (Edinb). 108(3):264-272.

Peona $\mathrm{V}$, et al. 2020. The avian $\mathrm{W}$ chromosome is a refugium for endogenous retroviruses with likely effects on female-biased mutational load and genetic incompatibilities. Philos Trans R Soc B. 376:20200186.

Procházka P, et al. 2011. Low genetic differentiation among reed warbler Acrocephalus scirpaceus populations across Europe. J Avian Biol. 42(2):103-113.

Roach MJ, Schmidt SA, Borneman AR. 2018. Purge Haplotigs: allelic contig reassignment for third-gen diploid genome assemblies. BMC Bioinformatics 19(1):460.

Røed T. 1994. "Rørsanger." In Norsk Fugleatlas, 382-383. Norsk Ornitologisk Forening.

Salewski V, Hochachka WM, Fiedler W. 2010. Global warming and Bergmann's rule: do central European passerines adjust their body size to rising temperatures? Oecologia 162(1):247-260.
Sambrook J, Fritsch EF, Maniatis T. 1989. Molecular cloning: a laboratory manual, 2nd ed. New York: Cold Spring, Harbor Laboratory Press.

Schaefer T, Ledebur G, Beier J, Leisler B. 2006. Reproductive responses of two related coexisting songbird species to environmental changes: global warming, competition, and population sizes. J Ornithol. 147(1):47-56.

Shafer ABA, et al. 2017. Bioinformatic processing of RAD-seq data dramatically impacts downstream population genetic inference. Methods Ecol Evol. 8(8):907-917.

Sigeman H, Strandh, M, et al. 2020. Genomics of an avian neo-sex chromosome reveals the evolutionary dynamics of recombination suppression and sex-linked genes. bioRxiv Preprint, Available from: 10.1101/ 2020.09.25.314088. Accessed September 17, 2021.

Sigeman H, Ponnikas S, Hansson B. 2020. Whole-genome analysis across 10 songbird families within Sylvioidea reveals a novel autosome-sex chromosome fusion. Biol Lett. 16(4):20200082.

Simão FA, Waterhouse RM, loannidis P, Kriventseva EV, Zdobnov EM. 2015. BUSCO: assessing genome assembly and annotation completeness with single-copy orthologs. Bioinformatics. 31(19):3210-3212.

Simonsen M, Mailund T, Pedersen CNS. 2008. Rapid neighbour-joining. In: Crandall KA, Lagergren J, editors. Algorithms in bioinformatics. WABI 2008. Lecture Notes in Computer Science. Vol 5251. Berlin, Heidelberg (Germany): Springer. p. 113-122. Available from: 10.1007/978-3-540-87361-7_10. Accessed September 17, 2021.

Skinner BM, Griffin DK. 2012. Intrachromosomal rearrangements in avian genome evolution: evidence for regions prone to breakpoints. Heredity (Edinb). 108(1):37-41.

Stanke M, Diekhans M, Baertsch R, Haussler D. 2008. Using native and syntenically mapped CDNA alignments to improve de novo gene finding. Bioinformatics 24(5):637-644.

Stokke B, et al. 2018. Characteristics determining host suitability for a generalist parasite. Sci Rep. 8(1):6285.

Stolt BO. 1999. The Swedish reed warbler Acrocephalus scirpaceus population estimated by a capture-recapture technique. Ornis Svecica 9:35-46.

Sætre CL, et al. 2017. Rapid adaptive phenotypic change following colonization of a newly restored habitat. Nat Commun. 8:14159.

The UniProt Consortium. 2021. UniProt: the universal protein knowledgebase in 2021. Nucleic Acids Res. 49:D480-D489.

Thorogood R, Davies NB. 2013. Reed warbler hosts fine-tune their defenses to track three decades of cuckoo decline. Evolution 67(12):3545-3555.

Thorogood R, Spottiswoode CN, Portugal SJ, Gloag R. 2019. The coevolutionary biology of brood parasitism: a call for integration. Philos Trans R Soc B. 374:20180190.

Vickery JA, et al. 2014. The decline of Afro-Palaearctic migrants and an assessment of potential causes. Ibis 156(1):1-22.

Vurture GW, et al. 2017. GenomeScope: fast reference-free genome profiling from short reads. Bioinformatics 33(14):2202-2204.

Associate editor: Bonnie Fraser 